Article

\title{
Wettability Investigations and Wet Transfer Enhancement of Large-Area CVD-Graphene on Aluminum Nitride
}

\author{
Marius Knapp ${ }^{1, *}$, René Hoffmann ${ }^{1}$, Volker Cimalla ${ }^{1}$ and Oliver Ambacher ${ }^{2}$ \\ 1 Fraunhofer Institute for Applied Solid State Physics IAF, Tullastraße 72, 79108 Freiburg, Germany; \\ rene.hoffmann@iaf.fraunhofer.de (R.H.); volker.cimalla@iaf.fraunhofer.de (V.C.) \\ 2 Department of Power Electronics, Institute of Sustainable Systems Engineering (INATECH), \\ University of Freiburg, 79085 Freiburg, Germany; oliver.ambacher@inatech.uni-freiburg.de \\ * Correspondence: marius.knapp@iaf.fraunhofer.de; Tel.: +49-761-5159-514
}

Received: 31 July 2017; Accepted: 15 August 2017; Published: 18 August 2017

\begin{abstract}
The two-dimensional and virtually massless character of graphene attracts great interest for radio frequency devices, such as surface and bulk acoustic wave resonators. Due to its good electric conductivity, graphene might be an alternative as a virtually massless electrode by improving resonator performance regarding mass-loading effects. We report on an optimization of the commonly used wet transfer technique for large-area graphene, grown via chemical vapor deposition, onto aluminum nitride (AlN), which is mainly used as an active, piezoelectric material for acoustic devices. Today, graphene wet transfer is well-engineered for silicon dioxide $\left(\mathrm{SiO}_{2}\right)$. Investigations on AlN substrates reveal highly different surface properties compared to $\mathrm{SiO}_{2}$ regarding wettability, which strongly influences the quality of transferred graphene monolayers. Both physical and chemical effects of a plasma treatment of AlN surfaces change wettability and avoid large-scale cracks in the transferred graphene sheet during desiccation. Spatially-resolved Raman spectroscopy reveals a strong strain and doping dependence on AlN plasma pretreatments correlating with the electrical conductivity of graphene. In our work, we achieved transferred crack-free large-area $\left(40 \times 40 \mathrm{~mm}^{2}\right)$ graphene monolayers with sheet resistances down to $350 \Omega /$ sq. These achievements make graphene more powerful as an eco-friendly and cheaper replacement for conventional electrode materials used in radio frequency resonator devices.
\end{abstract}

Keywords: graphene wet transfer; aluminum nitride; wettability; chemical vapor deposition; surface plasma treatment; graphene doping; CVD-graphene

\section{Introduction}

Graphene is a monolayer of hexagonal-oriented carbon atoms with outstanding electronic properties [1]. Its high electrical conductivity combined with its virtually massless character makes graphene interesting for electrodes in the field of acoustic devices operated at radio frequency (RF), such as surface and bulk acoustic wave resonators. Aluminum nitride (AlN) is a typically used material for such RF resonator devices [2,3]. Several groups as [4,5] could show that graphene reveals its potential as a conductive material to minimize the influence on the performance of resonator devices. For an unfailing use as an active electrode, graphene needs to exhibit low sheet resistances since the conductivity of the electrode material strongly affects the quality factor of a resonator $[6,7]$. These findings require high-quality large-area graphene on insulating substrates. Chemical vapor deposition (CVD) is the most promising technique for the synthesis of large-area graphene layers with a low sheet concentration of structural defects. Since the synthesis occurs on catalytic substrates [8], such as copper $(\mathrm{Cu})$ and nickel $(\mathrm{Ni})$, graphene needs to be transferred on substrates used for applications in 
electronic devices. In order to ensure a large-area continuous graphene sheet on the target substrate the transfer procedure within the graphene process, technology must be stable, avoiding the formation of broken bonds and cracks which decrease the electric performance of graphene. The standard transfer technique is wet transfer on silicon dioxide $\left(\mathrm{SiO}_{2}\right)$ substrates $[9,10]$. Sheet resistances $R_{S}$ down to $1.1 \mathrm{k} \Omega / \mathrm{sq}$ are achieved reproducibly [11]. Transferred graphene with $R_{S}=450 \Omega / \mathrm{sq}$ is commercially available from certain suppliers [12], which proves the mature process technology und usability of CVD graphene. In the field of RF microelectromechanical systems (RF MEMS), used e.g., in wireless communication, graphene is able to reveal both its conductive and two-dimensional character as an alternative electrode material in full strength. In this case, the transfer substrates are of piezoelectric nature, such as aluminum nitride (AlN). Graphene transfer on AlN is already adopted by many groups $[4,13,14]$, but a thorough examination and experimental optimization of the transfer method has not yet been shown. However, this is essential in order to improve the structural as well as the electric quality of graphene on the transfer substrate.

In this publication, we show an optimized wet transfer technique on AlN. As a two-dimensional conductive material, graphene can play an important role as an alternative electrode material regarding the elimination of viscous losses due to its virtually massless character. Using the wet transfer technique [15], the desiccation of graphene on the target substrate is the most critical process step. The interplay between baking after the process and the substrate's wettability strongly influences this desiccation procedure. In our work we found that hydrophobic surfaces tend to be responsible for additional strain-induced cracks in transferred graphene sheets. Therefore, the target surface has a great impact on the success of the wet transfer.

Commonly used silicon dioxide $\left(\mathrm{SiO}_{2}\right)$ as a target substrate is highly hydrophilic, therefore, no other surface treatment than standard substrate cleaning is necessary for a successful transfer [10]. However, the RF topic requires AlN, which is a relatively hydrophobic surface substrate, due to its piezoelectric character for RF resonator devices. The emerging cracks in the graphene monolayer result in very high sheet resistances and strongly decrease the performance of graphene as a conducting electrode material. Therefore, a surface plasma pretreatment process was developed to modify the surface energy of AlN. The increased wettability enables a more controlled and smooth desiccation and, concurrently, lower graphene sheet resistance. Our obtained results ensure an unchanged graphene layer quality during both synthesis and transfer and are therefore fundamental for ongoing research activities in the field of acoustic RF devices [16] using graphene as an active electrode on AIN. Depending on the plasma there is both a physical and a chemical effect on the surface properties which influences both the transfer and the performance of the fabricated device. The surface roughness of the substrate surface strongly influences the adhesion between graphene and substrate. The adhesion, represented by a van der Waals interaction, depends on the surface roughness due to a changing mean distance between graphene and the substrate, as Gao et al. showed [17]. Additionally, the chemical effect acts like a "cleaning" of the surface [18]. Our investigations show that using the chemical cleaning effect of plasma pretreatments to optimize the transfer process is beneficial. As a result, we are able to achieve large-area graphene transferred on AlN with $R_{S} \approx 350 \Omega /$ sq as a best value, which is strongly comparable to commercially available graphene on $\mathrm{SiO}_{2}$.

\section{Results}

\subsection{Wettability Investigations on AlN/Si Substrates}

Graphene transferred on an untreated AlN/Si substrate is shown in Figure 1. Optical microscope images (Figure 1b,c) indicate the cracks and gaps in the transferred graphene layer which stem from the desiccation process. The characteristic Raman 2D peak clearly reveals large graphene-free areas (Figure 1d). In contrast, graphene on $\mathrm{SiO}_{2}$ does not show cracks after transfer under the same transfer conditions (a). In order to investigate surface effects, $\mathrm{SiO}_{2}$ on $\mathrm{Si}$ and $\mathrm{AlN}$ on $\mathrm{Si}$ were characterized via atomic force microscopy (AFM). Figure 2 clearly shows that the $\mathrm{SiO}_{2}$ surface (a) is explicitly smoother 
than the AlN surface (b). The calculated root mean square (RMS) as a surface roughness parameter averages between $0.9 \pm 0.43 \mathrm{~nm}$ and $3.49 \pm 0.88 \mathrm{~nm}$ for $\mathrm{SiO}_{2}$ and $\mathrm{AlN}$, respectively (Figure 3i). The much lower roughness for $\mathrm{SiO}_{2}$ might be a first indication that the graphene transfer is less stable on the rougher AlN surface.
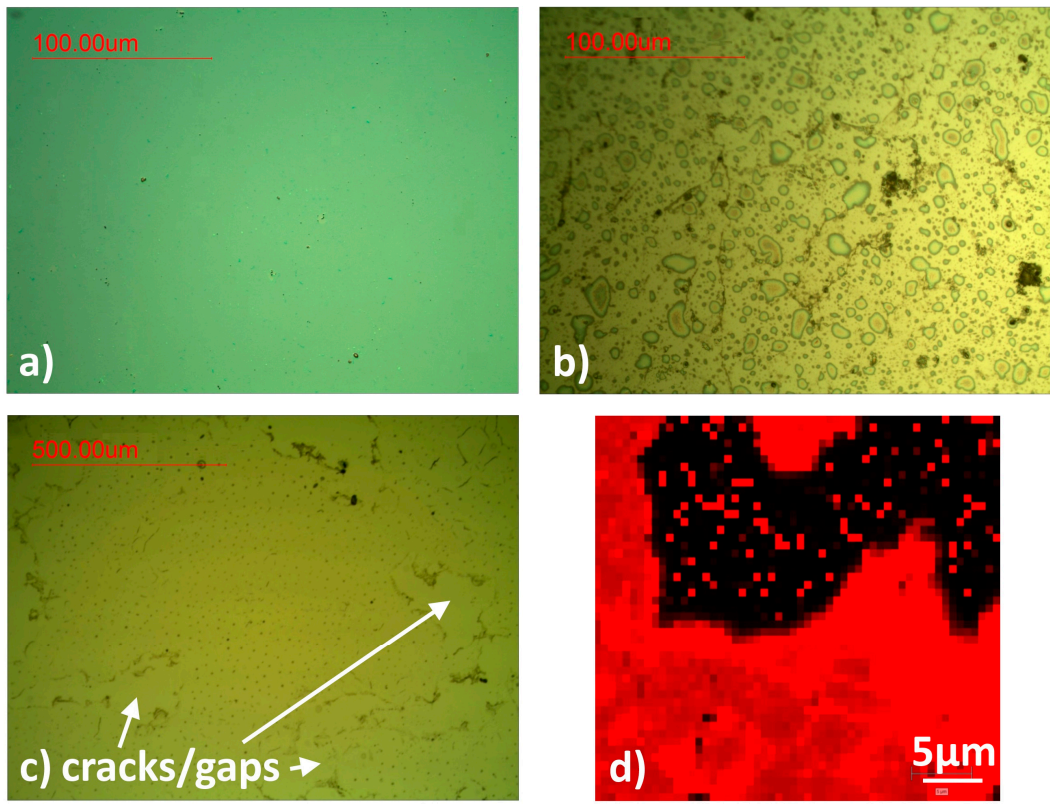

Figure 1. (a) Graphene on $\mathrm{SiO}_{2}$. (b,c) Graphene on untreated aluminum nitride (AIN). Circular structures show aqueous cavities (b). Arrows indicate areas without graphene (c,d) Raman 2D peak mapping of a randomly selected area with cracks/gaps (absent 2D peak, black) clearly visible and break the graphene layer (red).
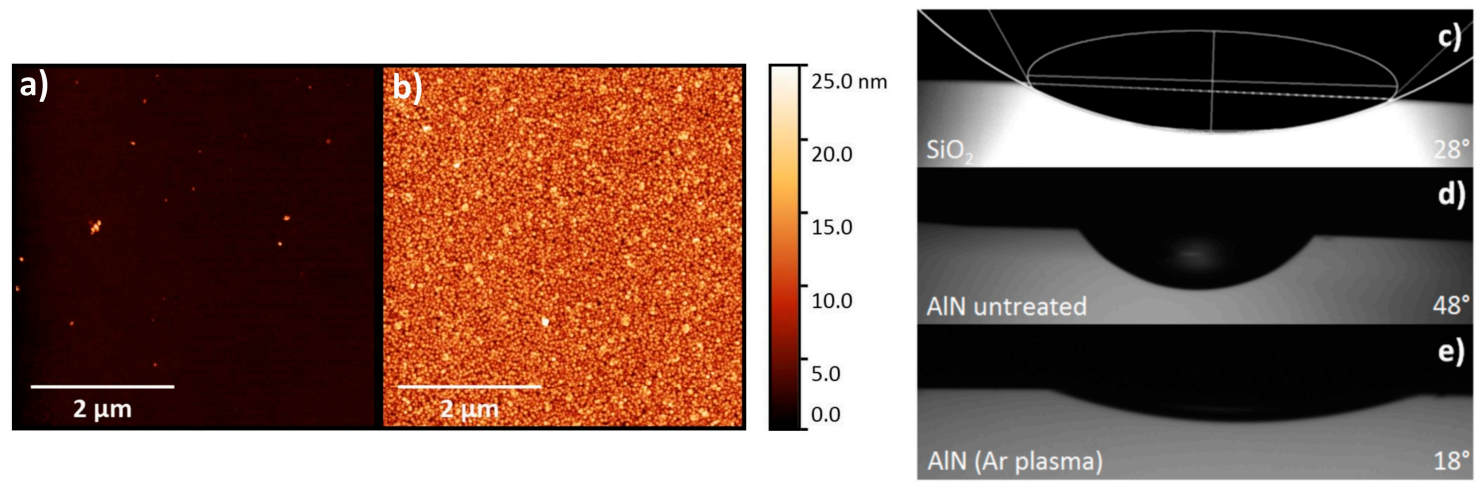

Figure 2. Atomic force microscopy (AFM) image of thermally oxidized $\mathrm{SiO}_{2}$ on $\mathrm{Si}$ (a) and $\mathrm{AFM}$ image of AlN sputtered on $\mathrm{Si}(\mathbf{b})$. (c-e) Optical images of a water droplet on different substrates-contact angles are obtained (d) via a circular and elliptical fit.

Since the wettability of the surface depends both on surface roughness and chemical conditioning, contact angle (CA) measurements were performed. According to the sessile drop method [19] very small water droplets of $10 \mu \mathrm{L}$ volume were used for the measurements, so that additional mass effects influencing the droplet shape can be neglected. Therefore, the contact angle can be obtained via a circular fit of the optical droplet image (Figure 2c). For untreated $\mathrm{SiO}_{2}$ and $\mathrm{AlN}$ on $\mathrm{Si}$ the contact angle is $28^{\circ}$ and $48^{\circ}$, respectively. Both AFM and CA measurement clearly reveal the different surface properties resulting in an unsatisfying graphene transfer on AlN. In a next step, AlN/Si samples were plasma treated in different ways before the transfer. The samples were directly exposed to $\mathrm{H}_{2}, \mathrm{~N}_{2}, \mathrm{O}_{2}$ and Ar plasmas. Figure 3 shows the related AFM images obtained for each AlN sample. 
In Figure 3, (a) shows an image of untreated AlN, (b) illustrates AlN after an $\mathrm{H}_{2}$ plasma, and (c), AlN after an Ar plasma. Figure 3e-g show a zoom-in to the corresponding samples. Ar plasma treatment significantly changes the topography of AlN whereas there is almost no change for $\mathrm{H}_{2}$ plasma pretreatment. The effect of $\mathrm{N}_{2}$ and $\mathrm{O}_{2}$ (not shown) is fairly similar as the one for Ar. Comparing the corresponding roughness parameters we obtained RMS values for $\mathrm{N}_{2}, \mathrm{O}_{2}$ and $\mathrm{Ar}(1.1-1.2 \mathrm{~nm})$, which are close to the one for $\mathrm{SiO}_{2}(0.9 \mathrm{~nm})$ (Figure 3i). For the $\mathrm{H}_{2}$ plasma the RMS value merely changes to $3.2 \mathrm{~nm}$.
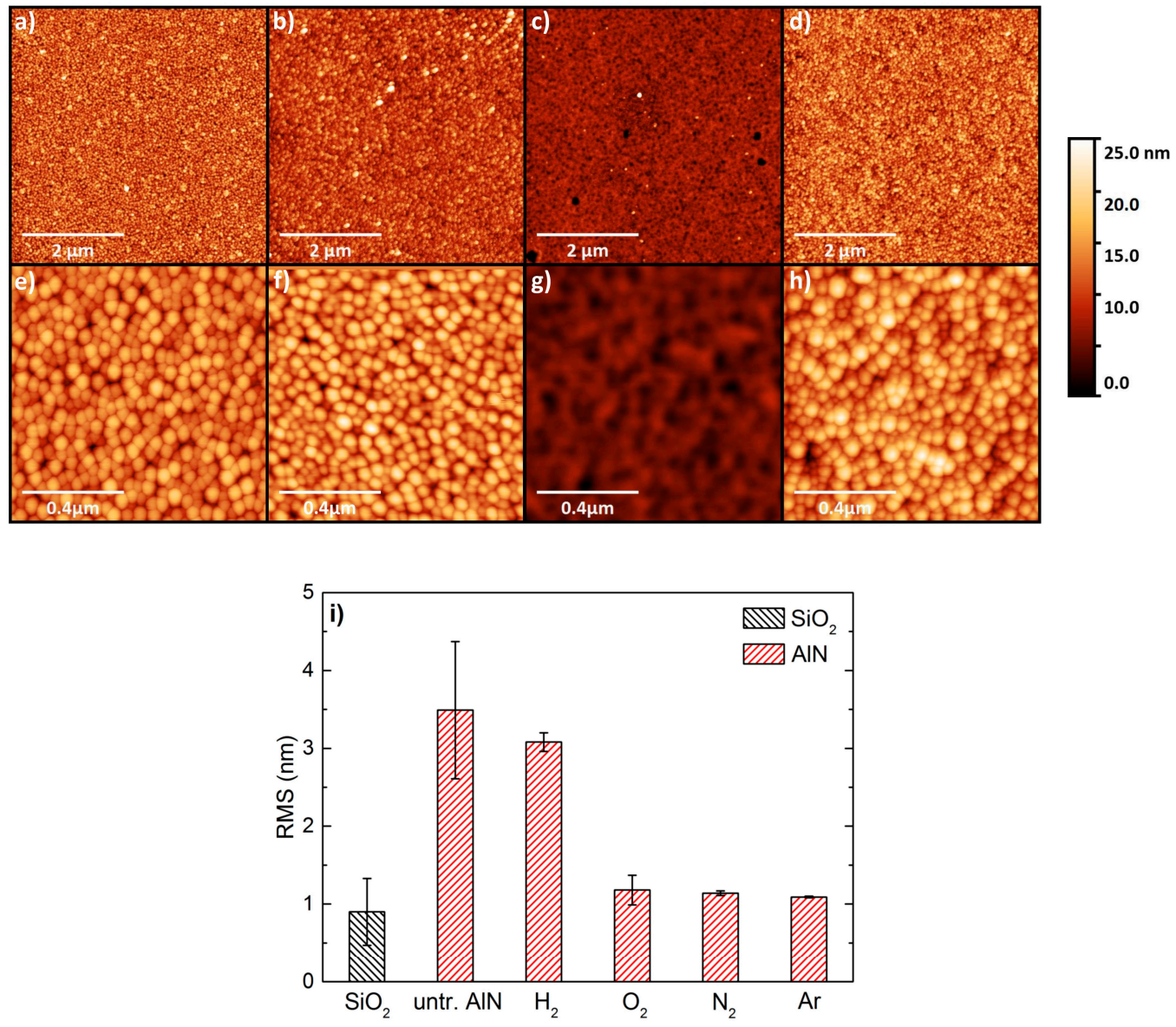

Figure 3. (a-h) AFM images of differently plasma pretreated AlN with different enlargement. As grown AlN on $\mathrm{Si}(\mathbf{a}, \mathbf{e}), \mathrm{H}_{2}$ plasma treatment $(\mathbf{b}, \mathbf{f})$ and Ar plasma treatment $(\mathbf{c}, \mathbf{g})$. An adjusted plasma process for of AlN on Bragg mirror structures (AlN-SMR), named as B2 in Section 2.2, reveals that the typically columnar structure of the AlN surface is preserved, which is shown exemplary in (d,h). (i) Comparison of RMS surface roughness parameter for $\mathrm{SiO}_{2}$ (black) and differently plasma treated $\mathrm{AlN} / \mathrm{Si}$ surfaces.

Corresponding CA measurements show a strong change in surface properties for the differently treated AlN samples. The obtained CA (Figure 4a) for measurements $2 \mathrm{~h}$ after pretreatment closely fit the RMS values. CA tremendously decreases to approx. $18^{\circ}$ for $\mathrm{N}_{2}, \mathrm{O}_{2}$ and Ar plasma pretreatments. In general, the contact angle between a fluid and a solid is described by the Young equation [20]:

$$
\gamma_{S A}-\gamma_{S L}-\gamma_{L A} \times \cos \theta=0
$$

with the surface tension $\gamma$ and the corresponding indices S (solid), A (air) and L (liquid) for the phases. $\theta$ is called the Young or chemical angle which describes the contact angle for a perfectly flat surface. 
According to the Wenzel model which describes homogenous wetting regimes, surface roughness changes the contact angle via

$$
\cos \theta^{*}=r \times \cos \theta
$$

where $\theta^{*}$ is the corrected contact angle including the roughness parameter $r$, which is the ratio between the real and the projected solid surface area [21]. The Wenzel model establishes the connection of surface roughness on surface wettability. Higher roughness leads to lower wettability. Heavy atoms like $\mathrm{N}_{2}, \mathrm{O}_{2}$ and Ar physically damage the AlN structure and smoothen its surface, whereas $\mathrm{H}_{2}$ has almost no major physical impact on AlN due to its low atomic weight. The surface damage is clearly seen in Figure $3 \mathrm{~g}$. The typically pebble-like structure for sputtered AlN is destroyed, although the surface is smoother than without any plasma treatment.
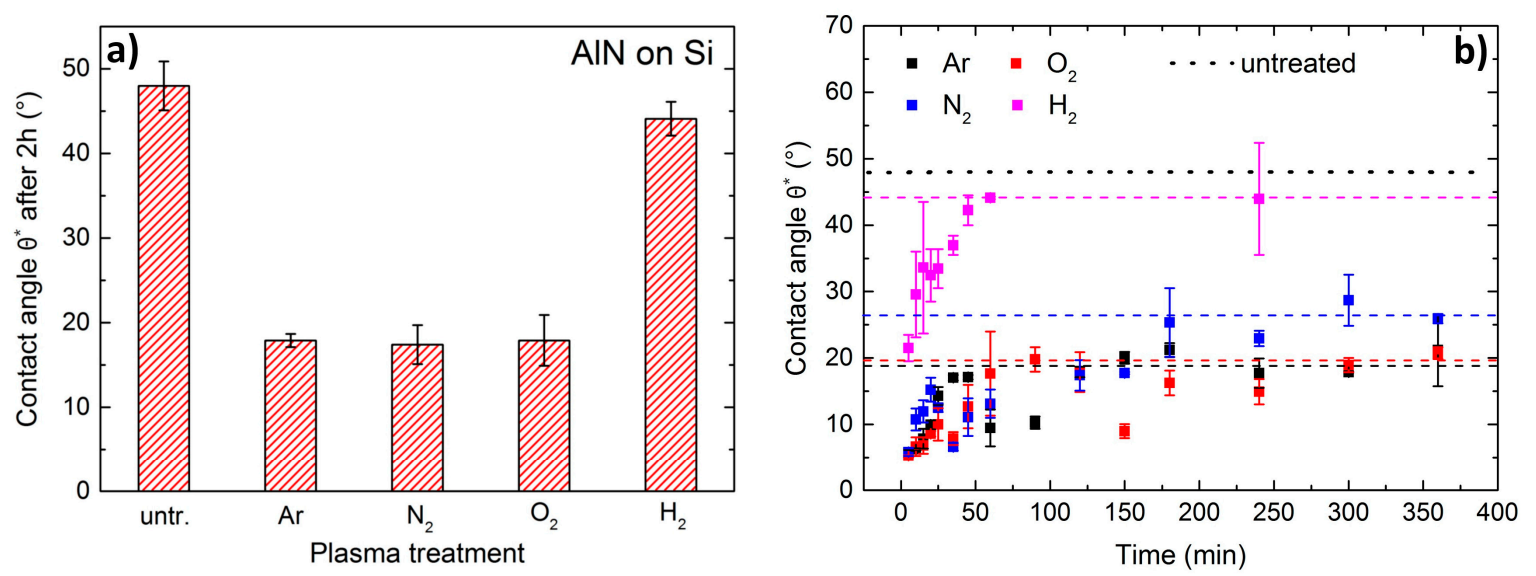

Figure 4. (a) Contact angle (CA) for all pretreatment variations measured after $2 \mathrm{~h}$. (b) Time-dependent contact angle measurements for $\mathrm{Ar}, \mathrm{O}_{2}, \mathrm{~N}_{2}$ and $\mathrm{H}_{2}$ plasma pretreatment of AlN on Si. The black dotted line represents $\mathrm{CA}$ for untreated $\mathrm{AlN}$ on $\mathrm{Si}$. A recovery effect is strongly visible.

Apart from this physical smoothing which influences the substrates wettability, there is another important effect responsible for the surface properties. Plasma treatment also leads to a change in the chemical composition of the surface. For all plasmas there is a cleaning effect of the surface. Contamination atoms like carbon and other carbonic composites, which tend to be hydrophobic, are both physically and chemically removed. These composites can readsorb on the AlN surface after the plasma pretreatment [22], which indicates a time-dependent recovery effect of the AlN substrate's wettability.

In addition to this plasma cleaning effect, Sönmez et al. [18] explore another impact of different plasma gases on stainless steel. In case of an $\mathrm{O}_{2}$ plasma, there is an oxidation effect of the surface which in turn becomes more hydrophilic due to a smoothening. A certain amount of residual $\mathrm{O}_{2}$ within the reactor chamber might enable oxidizing reactions which results also in a smoothening of the AlN surface. A nanolayer of aluminum oxide allotropes directly increases the wettability. Depending on the fabricated application one needs to keep in mind that oxidation might cause undesired side effects on the device performance (see Section 2.2). Dehydration effects, e.g., during the wet transfer, might decrease the wettability again.

Physical damage in combination with $\mathrm{N}_{2}$ or $\mathrm{H}_{2}$ plasma might also form nitrate composites, such as $\mathrm{Al}\left(\mathrm{NO}_{3}\right)_{3}$ and $\mathrm{NH}_{4} \mathrm{NO}_{3}$, due to the aforementioned, non-negligible amount of residual oxygen in the plasma chamber. Despite their negligible effect in physical surface smoothening, $\mathrm{H}_{2}$ atoms might create labile hydroxide compounds with the assistance of background oxygen, such as $\mathrm{Al}(\mathrm{OH})_{3}$. These chemical reactions can also activate the AlN surface due to the availability of aluminum atoms in the substrate material. The creation of nitrate components as intermediate products of chemical reactions increase, as well as oxides, the surface energy i.e., wettability [22]. Nitrate components 
are unstable and tend to degrade very soon and also dehydration takes place right after the plasma treatment. On the contrary an oxidized layer on the AlN surface can endure. All of these described effects can strongly influence the total surface wettability and might explain the recovery effect below.

The "cleaning" phenomenon of the removal of surface contaminations can be seen in Figure $4 \mathrm{~b}$. Time-dependent CA measurements obviously show a recovery effect. It is clearly visible that there is a strong reduction in $\theta^{*}$ for all applied plasmas within the first minutes after plasma exposure. This drop in CA is rapidly recovered within one hour after pretreatment. The recovery effect is quickest for $\mathrm{H}_{2}$ plasma. There is a specific saturation $\mathrm{CA}$ value for each of the plasma pretreatments, which is time-independent and averages between $44^{\circ}$ for $\mathrm{H}_{2}$ and $18^{\circ}$ for $\mathrm{N}_{2}, \mathrm{O}_{2}$ and $\mathrm{Ar}$ (dashed lines). Hence these maximum values of $\theta^{*}$ for $t>2 \mathrm{~h}$ for different plasmas represent the ones shown in Figure $4 \mathrm{a}$. There exists an explicitly noticeable hydrophobic recovery with increasing time. By repeating the plasma pretreatment, the time-dependent change of the contact angles can be obtained at every measurement cycle. The difference in recovery might be explained with the above mentioned chemical reactions. For $\mathrm{Ar}, \mathrm{N}_{2}$, and $\mathrm{O}_{2}$ plasma several oxidation reactions superpose the cleaning effects, whereas for $\mathrm{H}_{2}$ plasma a stable oxidation apparently does not take place. For this purpose a profound analysis of the chemical compounds on the AlN surface after plasma treatment needs to be carried out, this is not within the scope of this publication.

In a first summary we can conclude that plasma pretreatments impact the surface both physically and chemically, whereas a chemically activated surface shows an aging effect. Transfer experiments indicate that already a physical impact on the AIN surface resulting in as little as $\theta^{*}<20^{\circ}$ improves the quality of transferred graphene. The Raman D/G ratio decreases below 0.2 and the crack density can be lowered significantly. As shown later on this influences directly the graphene sheet resistance.

\subsection{Wettability Investigations on BAW-SMR Substrates}

Since we are interested in graphene as a top electrode for resonator devices, we adapted the transfer process including the plasma treatment on solidly mounted bulk acoustic wave AlN resonator structures (BAW-SMR, described in detail in Section 4.1) B1 and B2, which were grown on a Bragg reflector structure and different bottom electrodes. In this case, an increase in wettability without a change in surface roughness and stable oxide allotropes is preferable, to avoid a potentially different performance characteristic of the resonator devices due to a changed AlN surface roughness. This is important regarding the performance characteristics of actual devices, such as BAW resonators, where a structural change of the surface can influence future device functionality. For this reason we adjusted the plasma treatment in a further experimental step by reducing power and ambient pressure during the plasma process to lower the direct plasma exposure of the AlN substrates. In this case the change in wettability occurs only because of the chemical conditioning of the surface and not as a consequence of physical damage of the AlN surface. Monolayer graphene was therefore transferred on AlN grown on a material stack working as a solidly mounted resonator device (BAW-SMR). Figure 5a,b show RMS obtained from AFM images and CA values, respectively, for both resonator structures. $\mathrm{SiO}_{2}$ and AlN on Si are shown as a reference. Although the two samples differ in their surface roughness $\left(\mathrm{RMS}=4.4 \pm 0.7 \mathrm{~nm}(1.8 \pm 0.2 \mathrm{~nm})\right.$ for B1 (B2)) the CA is remarkably high for B2 $\left(63 \pm 6^{\circ}\right)$. When an Ar plasma is applied on both samples RMS values do not change (AFM image in Figure 3d,h), but CA strongly decreases to $19-22^{\circ}$ (Figure 5 c). The comparably large error bars result from a relatively fast recovery effect (CA measurements are performed within $30 \mathrm{~min}$ after plasma treatment). Hence both a physical damage and stable oxidation effects can be ruled out. These experimental results demonstrate that wettability manipulations of the AlN surface are possible without physical surface smoothening which might deteriorate other device properties. For this, the transfer step needs to occur directly after the plasma pretreatment. 

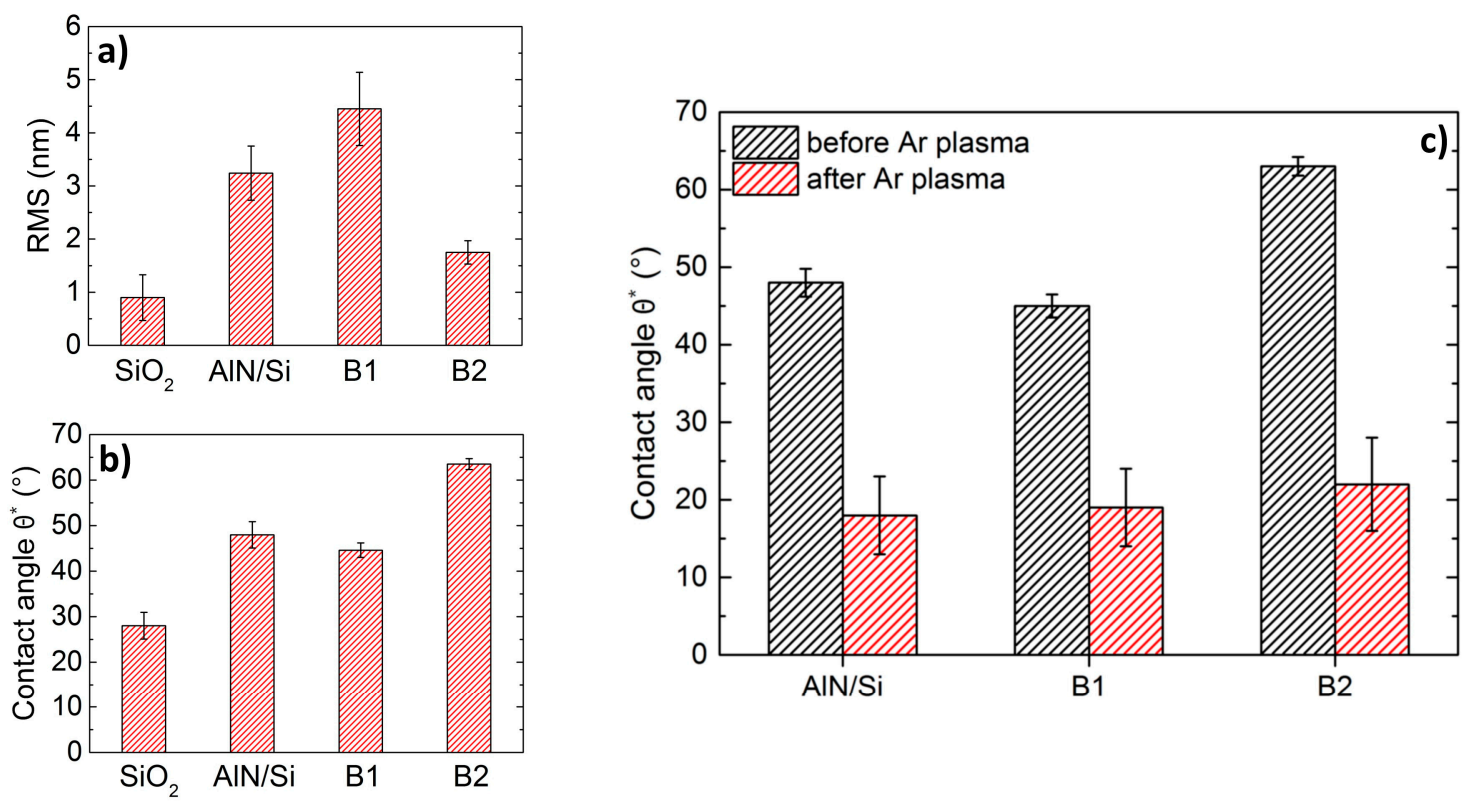

Figure 5. (a) Comparison of roughness values (RMS) obtained from AFM images for AlN/Si, $\mathrm{B} 1(\mathrm{AlN} / \mathrm{TiAlCuW})$ and $\mathrm{B} 2(\mathrm{AlN} / \mathrm{MoAlN})$ substrates, $\mathrm{SiO}_{2}$ as a reference. (b) Comparison of corresponding CA obtained by dropping water droplets on the surface. (c) Comparison of CA before and 30 min after Ar plasma treatment.

\subsection{Strain and Doping Investigations via Spatially-Resolved Raman Spectroscopy}

Additionally, the strain distribution across the transferred graphene layer was investigated to further understand the effect of plasma pretreatment. Using spatial Raman spectroscopy analysis of the $2 D$ and $G$ peak positions, $\omega_{2 D}$ and $\omega_{G}$, respectively, one is able to draw conclusions about strain and charge carrier density due to the strain and doping sensitivity of the Raman G and 2D modes [23]. In Figure $6, \omega_{2 D}$ versus $\omega_{G}$ is plotted for graphene on differently pretreated AlN substrates. The red (blue) line shows different levels of hole doping (strain). The slopes are obtained from [23], as

$$
\begin{aligned}
\left(\frac{\Delta \omega_{2 D}}{\Delta \omega_{G}}\right)_{\varepsilon} & =2.2 \pm 0.2 \\
\left(\frac{\Delta \omega_{2 D}}{\Delta \omega_{G}}\right)_{n}^{\text {hole }} & =0.70 \pm 0.05 .
\end{aligned}
$$

According to the authors, electron doping would show a nonlinear slope and in this case Equation (4) would not hold. Hall measurements of graphene on AlN showed hole doping, which allows the use of the aforementioned equations. Das et al. $[24,25]$ revealed a $\omega_{2 D}$ dependency on hole doping as $\Delta \omega_{2 D} / \Delta n=-1.04 \mathrm{~cm}, n$ in terms of $10^{12} \mathrm{~cm}^{-2}$. Yoon et al. [26] calculate the dependence of strain on a shift of $\omega_{G}$ as $\Delta \omega_{G} / \Delta \varepsilon=-23.5 \mathrm{~cm}^{-1} / \%$. These two relations lead to a direct identification of strain and doping of graphene on the differently pretreated AlN substrates. 

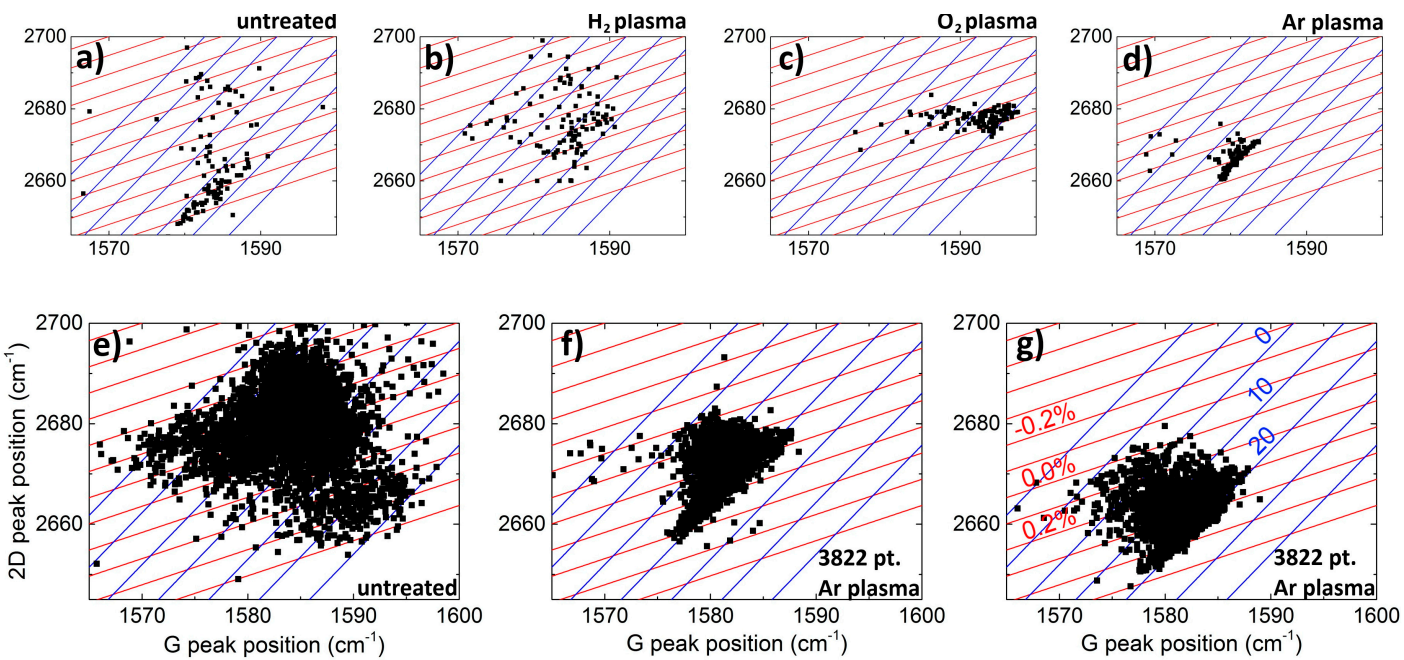

Figure 6. Raman spectroscopy measurements of graphene on AlN-2D peak position $\left(\omega_{2 D}\right)$ versus G peak position $\left(\omega_{G}\right)$. Line scan (121 data points) for differently pretreated AlN/Si substrates (a-d). Raman map of graphene on AlN (3822 data points, $30 \times 50 \mu \mathrm{m}^{2}$ area) for untreated AlN/Si (e), Ar plasma pretreated AlN/Si substrate (f) and Ar plasma pretreated B1 substrate (g). Shift in doping visible due to addition of $\mathrm{AuCl}_{3}$ during etching process. Level curves for compressive and tensile strain (blue line, in \%) and for doping concentration $n$ (red line, in $10^{12} \mathrm{~cm}^{-2}$ ).

In Figure 6, a Raman line scan of 121 data points is plotted (a-d). $\omega_{2 D}$ versus $\omega_{G}$ reveals a significant change for plasma pretreated AlN on Si. Strain and doping levels are labelled in Figure $6 \mathrm{~g}$ and hold for each of the graphs. A rather linear dependency is visible for both $\mathrm{O}_{2}$ and Ar plasmas, whereas there is no dependency identifiable for untreated $\mathrm{AlN}$ and $\mathrm{AlN}$ pretreated with an $\mathrm{H}_{2}$ plasma. Graphene on $\operatorname{Ar}\left(\mathrm{O}_{2}\right)$ plasma pretreated AlN shows dominantly tensile strain between $0.1-0.3 \%(0.1-0.2 \%)$; the doping is significantly lower for the Ar plasma $\left(n=2 \times 10^{11}-1 \times 10^{13} \mathrm{~cm}^{-1}\right)$ compared to the $\mathrm{O}_{2}$ plasma $\left(n=2 \times 10^{13}-4 \times 10^{13} \mathrm{~cm}^{-1}\right)$. The same result is obtained for $\mathrm{N}_{2}$ (not shown). We can conclude that variations of strain and doping are strongly reduced applying an $\mathrm{N}_{2}, \mathrm{O}_{2}$ or Ar plasma instead of an $\mathrm{H}_{2}$ or no plasma pretreatment at all. To obtain a greater spatial distribution map measurements were done, shown in (e-g) for untreated and Ar plasma treated AlN substrate (AlN/Si and B1). Due to observable D peak intensities for graphene on untreated AIN (e) information about actual doping concentrations cannot be extracted due to overlapping effects in the Raman shifts. However, a clear reduction of strain variation is apparent for the Ar plasma for both AlN/Si and B1 (f,g). The same results hold for B2 (not shown). This indicates that the transfer-induced strain results not from surface roughness itself but from wettability effects on the graphene sheet during transfer. For the transfer of graphene on $\mathrm{B} 1$ we added exemplary $\mathrm{AuCl}_{3}$ during the $\mathrm{Cu}$ etching, which results in an additional doping of the transferred layer. This is directly visible in a shift of the $2 \mathrm{D} / \mathrm{G}$ ratio $(\mathrm{g}) . \mathrm{AuCl}_{3}$ might play an important role regarding the decrease of sheet resistance due to the doping effect. This will be investigated further.

\section{Discussion}

In order to quantify the enhanced graphene quality due to the applied plasma treatments the samples were investigated via optical microscopy and Raman spectroscopy. Figure 7 shows an obvious improvement of the graphene wet transfer. Cracks and large-area gaps are avoided and the graphene sheet is transferred completely (microscopic images). Raman spectroscopy shows a clear quality enhancement. The defect-related D peak $\left(1350 \mathrm{~cm}^{-1}\right)$ vanishes almost completely, whereas the $2 \mathrm{D} / \mathrm{G}$ intensity ratio increases up to 2.6. The $2 \mathrm{D}$ peak lies at $2772 \mathrm{~cm}^{-1}$. Both indicate the existence of mostly monolayer graphene on AIN [27]. This result directly corresponds with the reduction of strain variation after Ar plasma treatment (Section 2.3). Non-uniform in-plane strain in graphene raises the probability of creating cracks and wrinkles in the layer. 

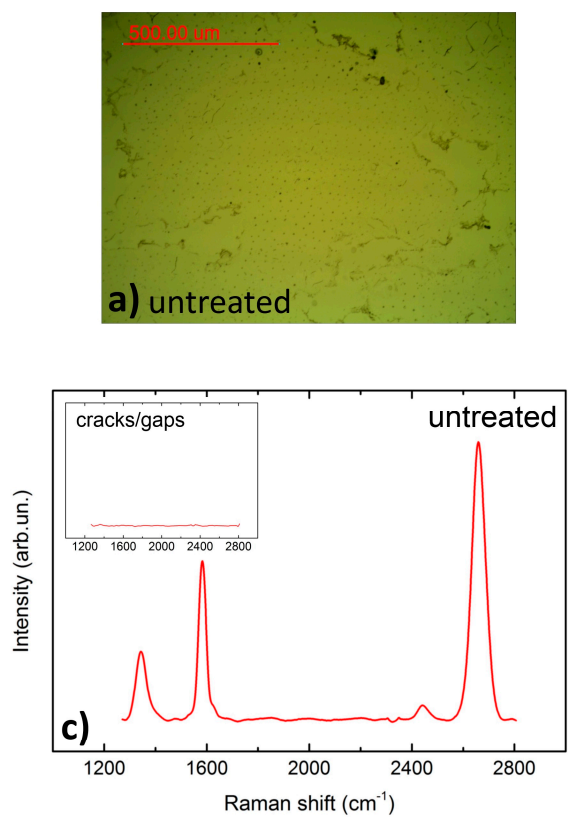
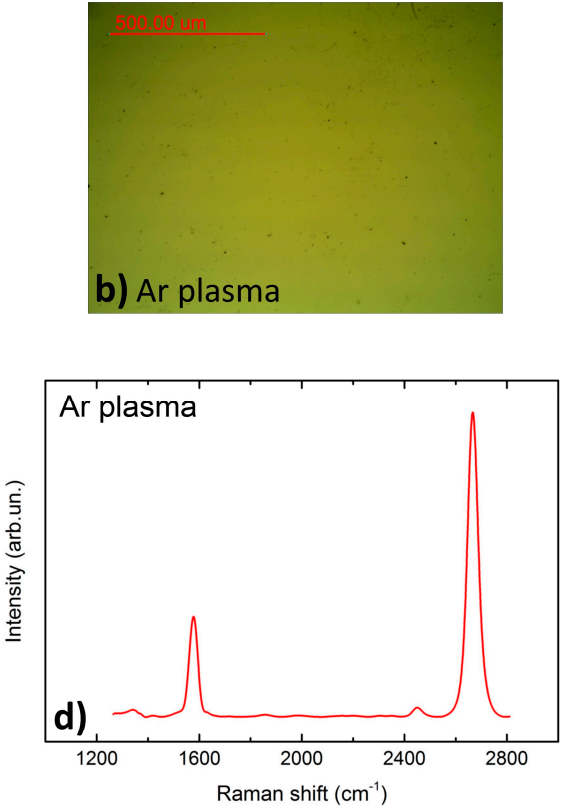

Figure 7. Comparison of transferred graphene on untreated AlN (a,c) and Ar plasma treated AlN (b,d). Optical microscope images show avoidance of cracks/gaps in the graphene sheet for Ar plasma treated AlN (b). Raman spectroscopy image shows an average of 121 measurements (line scan); inset in (c) shows no graphene Raman signal in gap areas. D peak is strongly reduced for Ar plasma treated AlN substrate $(\mathbf{d})$.

The obtained quality improvement is directly proven via four-point measurements. Due to plasma pretreatment, the sheet resistance $R_{S}$, shown in Figure 8, drops almost one order of magnitude for all applied plasmas (from $22 \mathrm{k} \Omega / \mathrm{sq}$ to $<5 \mathrm{k} \Omega / \mathrm{sq}$ ). The high error bars, especially for the untreated AlN samples, result from the probe distance of $1 \mathrm{~mm}$, which makes resistance measurements strongly dependent on the created gaps. According to Raman measurements, small graphene domains might have slightly lower sheet resistances. The most reliable results are achieved for Ar plasma pretreatment. Here, $R_{S}$ constantly reaches values below $1 \mathrm{k} \Omega / \mathrm{sq}$. Best values were obtained as $R_{S}=350 \Omega / \mathrm{sq}$. Sheet resistance measurements were homogenous on an area of $40 \times 40 \mathrm{~mm}^{2}$. So far, we can exclude the effect of induced additional strain due to doping, as shown in Figure 6g. Therefore, a further reduction of $R_{S}$ seems to be promising. Additional experiments on this topic still need to be carried out.

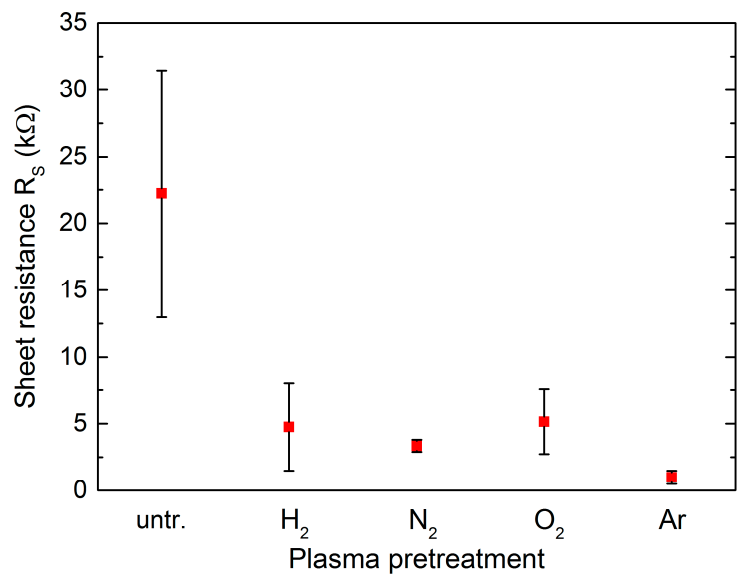

Figure 8. Sheet resistance of graphene monolayers transferred on $\mathrm{AlN} / \mathrm{Si}$ as a comparison for different surface plasma pretreatment. Relatively high measurement errors obtained for untreated AlN due to $1 \mathrm{~mm}$ probe distance of the measurement setup. 
As a conclusion, our experiments clearly reveal that the most delicate process step of the wet transfer technique is the graphene's desiccation on the target substrate. Figure 9a shows the wettability concept on different substrates. Due to their high wettability, hydrophilic surfaces (such as $\mathrm{SiO}_{2}(1)$ ) ensure a continuous desiccation via capillary forces in between the substrate and the graphene/PMMA (Poly (methyl methacrylate)) whereas hydrophobic surfaces (such as AlN (2)) form water droplets instead. These droplets either remain in between the substrate and the graphene layer (1) or they break the graphene layer for sublimation, creating cracks and discontinuities (2). As shown experimentally in this work, additional strain is applied on the transferred graphene sheets, which critically influences the sheet resistance $R_{S}$ of the graphene. The underlying effect of hydrophobic surfaces is the concept of surface and interfacial energies, which we identified indirectly via CA measurements. Hydrophobic surfaces feature a low surface energy and hydrophilic surfaces feature a high surface energy [28]. Because of the high surface energy of water due to its polar character, the surface energy of AlN must be increased. In our experiments, this was done by plasma treatment. Thinking of a further optimization of graphene as an electrode material, a stacking of several graphene sheets transferred on top of each other might be reasonable to further decrease $R_{S}$. Problems regarding the transfer of graphene onto graphene will occur due to its extremely hydrophobic behavior. CA measurements revealed $\theta^{*}$ to be very close to $90^{\circ}$ (Figure $9 \mathrm{~b}$ ). This means that the topic of surface wettability will continue to arise with respect to the fabrication of high-quality large-area graphene multilayers. Furthermore, an area-increase of transferred graphene towards wafer-size makes the desiccation process even more challenging.
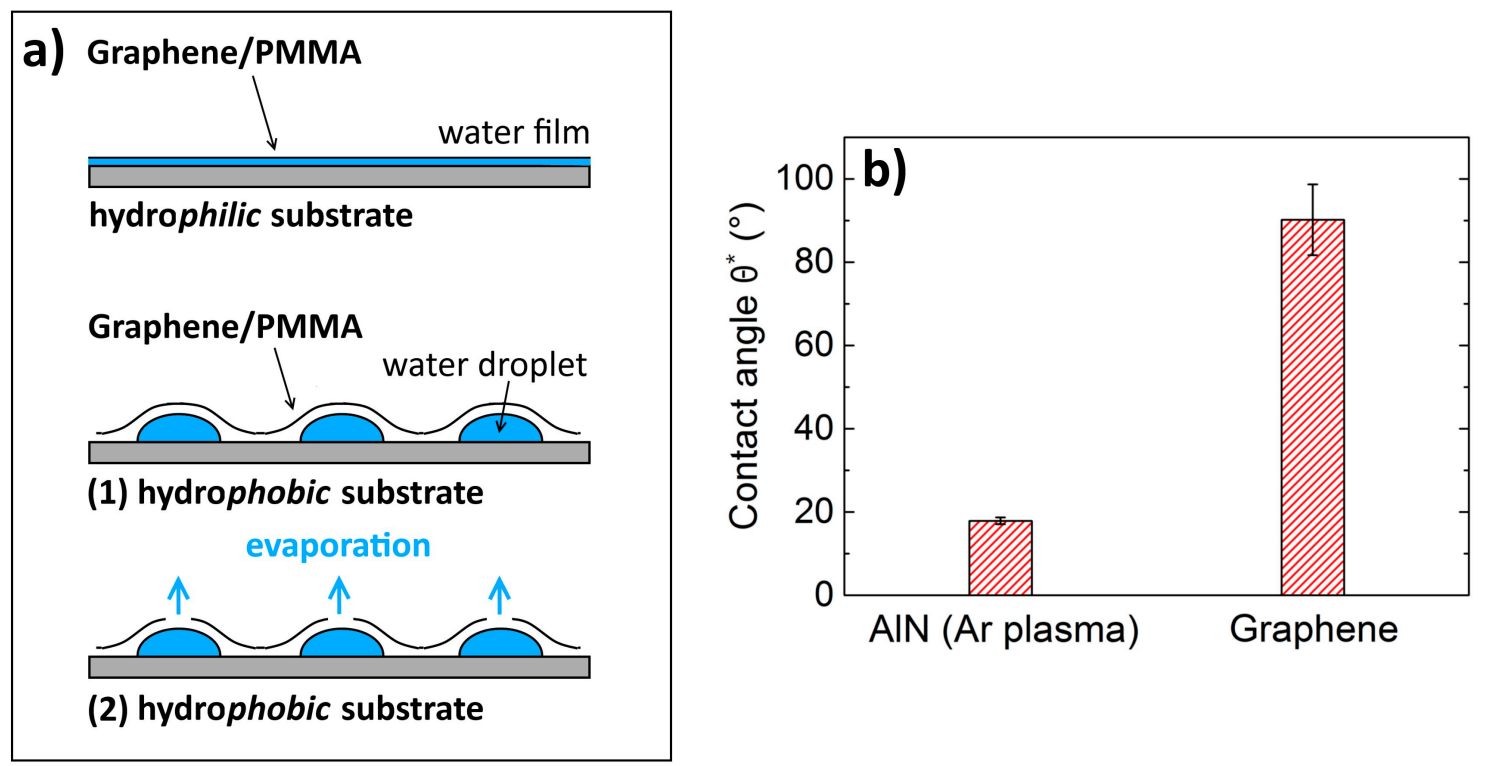

Figure 9. (a) Schematic figure of the wettability concept. Different wettability for hydrophobic and hydrophilic target surfaces influence the desiccation process resulting in gaps/cracks and non-uniform strain within the transferred graphene layer. (b) Comparison of CA for Ar-treated AIN and a pure graphene surface.

\section{Materials and Methods}

\subsection{Materials}

In this work, the synthesis of a graphene monolayer was achieved via chemical vapor deposition (CVD), a mature and commonly applied technique of growing graphene [29], on copper (Cu) foil (25 $\mu \mathrm{m}$ thickness, 99.8\% purity, Alfa Aesar, Haverhill, MA, USA) used as a catalytic metal substrate in a cold wall low-pressure reactor (BlackMagic Pro, Aixtron, Herzogenrath, Germany) using methane 
and hydrogen as precursor and carrier gas, respectively (flow ratio $\mathrm{CH}_{4} / \mathrm{H}_{2}=25 \%, 1600 \mathrm{~Pa}, 980{ }^{\circ} \mathrm{C}$, 30 min deposition time).

Primarily, AlN substrates were investigated which are fabricated via sputtering ( $2 \mu \mathrm{m}$ AlN on $500 \mu \mathrm{m}$ silicon (AlN/Si, 4" wafer size)). The AlN wafers were cut into $20 \times 20 \mathrm{~mm}^{2}$ pieces for $10 \times 15 \mathrm{~mm}^{2}$ graphene transfers. Solidly mounted BAW resonators (BAW-SMR) with sputtered AlN as a piezoelectric layer (3" wafer size, $1.7 \mu \mathrm{m}$ AlN on TiAlCuW (named B1) and $1.5 \mu \mathrm{m}$ AlN on MoAlN (B2)) were used for $40 \times 40 \mathrm{~mm}^{2}$ graphene transfers (Table 1). Both AlN/Si and AlN-SMR wafers were fabricated by our project partner within the Graphene Flagship and provided to us for transfer experiments.

Table 1. Overview about used AIN samples for the graphene wet transfer.

\begin{tabular}{ccc}
\hline Substrate & Substrate Size & Graphene Layer Size \\
\hline AlN/Si & $20 \times 20 \mathrm{~mm}^{2}$ & $10 \times 15 \mathrm{~mm}^{2}$ \\
B1 (AlN/TiAlCu) & 3" wafer & $40 \times 40 \mathrm{~mm}^{2}$ \\
B2 (AlN/MoAlN) & 3" wafer & $40 \times 40 \mathrm{~mm}^{2}$ \\
\hline
\end{tabular}

\subsection{Methods}

The graphene on $\mathrm{Cu}$ foil was routinely investigated by a Raman spectroscopy line scan of 121 data points (InVia Raman microscope, $532 \mathrm{~nm}$ excitation laser, grating 1800 lines/mm, Renishaw, Gloucestershire, UK), since it is an approved, easily applicable, non-destructive characterization method [30]. The representative 2D/G peak intensity ratio of reproducibly above 2.5 and the FWHM (Full Width at a half Maximum) of the 2D peak of $<30 \mathrm{~cm}^{-1}$ clearly identifies this as a monolayer structure $[27,31]$. An almost imperceptible defect peak $(D / G$ ratio $<0.15)$ supports the conclusion that our synthesized material is a low-defect monolayer of graphene.

After CVD synthesis, the graphene monolayers were transferred on differently pretreated AlN substrates using the wet transfer technique (Figure 10), described in detail in [15], in a slightly aligned procedure. Using a PMMA (Poly (methyl methacrylate)) coating as a protection layer of the graphene/ $\mathrm{Cu}$ stack the copper foil was etched off with an aqueous ammonium persulfate solution $\left(\left(\mathrm{NH}_{4}\right)_{2} \mathrm{~S}_{2} \mathrm{O}_{8}, 0.5 \mathrm{~mol} / \mathrm{L}\right)$. This turned out to result in a cleaner interface between $\mathrm{AlN}$ and graphene after transfer due to lower formation of precipitates and chemical byproducts than commonly used etching solvents like iron chloride $\left(\mathrm{FeCl}_{3}\right)$ and iron nitrate $\left(\mathrm{Fe}\left(\mathrm{NO}_{3}\right)_{3}\right)$. Wu et al. [32] suggest $\left(\mathrm{NH}_{4}\right)_{2} \mathrm{~S}_{2} \mathrm{O}_{8}$ as an etching solvent since it avoids additionally induced Fe compounds during etching, which reduce the quality of the grown graphene. The PMMA/graphene stack was then deposited onto different AlN target substrates. Before the graphene transfer, the AlN substrates were exposed to different plasmas $\left(\mathrm{H}_{2}, \mathrm{~N}_{2}, \mathrm{O}_{2}\right.$ and $\left.\mathrm{Ar}, 10 \mathrm{~min}\right)$ in order to vary the surface conditions regarding surface energy and roughness. The $\mathrm{SiO}_{2}$ substrates received no plasma treatment and were used as reference samples. For comparison purposes, graphene transferred on untreated AlN was also investigated. Plasma pretreatments of AlN samples were done in a direct current (DC) plasma reactor (Leybold, 100-300 W, $9 \times 10^{-3}-5 \times 10^{-2} \mathrm{mbar}, 10 \mathrm{~min}$ exposure time). The wettability of the AlN sample was characterized via contact angle measurements using the sessile drop method [33] in a self-construction experimental setup. Here, a precise test droplet of deionized (DI) water $(10 \mu \mathrm{L})$ was dispensed on the AlN surface. The obtained video image of the droplet was fitted (ImageJ, image processing software, contact angle plugin) to extract the contact angle. The AlN surface roughness was investigated with atomic force microscopy (AFM; Nanowizard III, JPK instruments, Berlin, Germany). In addition, the sheet resistance of the transferred graphene was measured with the four-point method (Loresta-GX MCP-T700, $1 \mathrm{~mm}$ probe distance, Mitsubishi Chemical Analytech, Yamato, Japan). 


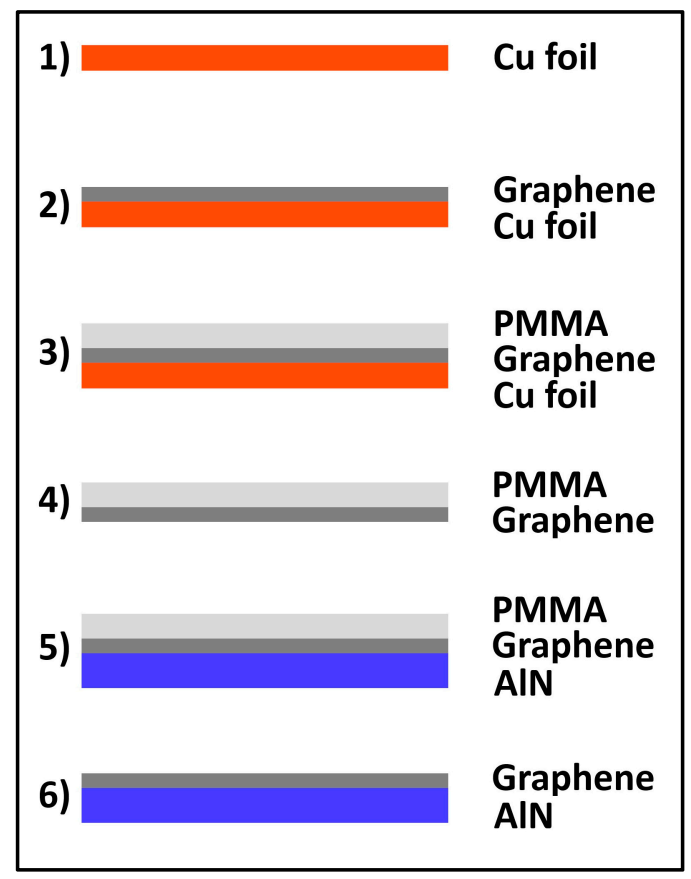

Figure 10. Schematic illustration of standard wet transfer process on aluminum nitride (AlN) after graphene chemical vapor deposition (CVD) growth.

\section{Conclusions}

The wet transfer of CVD graphene monolayers onto AlN was investigated. We identified a strong dependence of the wettability of AlN surfaces on different plasma pretreatments due to both a physical and a chemical effect on the AlN surface. AFM images and contact angle measurements reveal a change of surface properties resulting in spatially lower strain variation and a tremendous reduction of layer cracks and gaps for $\mathrm{N}_{2}, \mathrm{O}_{2}$ and Ar plasma pretreatments. Focusing on the chemical "cleaning" of the surface leads to a temporal but strong increase of wettability, which enables a defect-free graphene transfer without changing the AlN's surface morphology. Sheet resistance measurements show a direct effect of this optimization regarding sheet conductivity. $R_{S}$ can reproducibly be reduced below $1 \mathrm{k} \Omega / \mathrm{sq}$ for transferred graphene with the size of $40 \times 40 \mathrm{~mm}^{2}$ and an Ar plasma pretreatment.

With this new knowledge, the wet transfer method for the assembly of multilayer graphene sheets for further sheet resistance reduction should be investigated further. A sensitive adjustment of the surface properties of graphene regarding its strong hydrophobic behaviour is essential to obtain even lower sheet resistances. Furthermore, the effect of substrate surface pretreatment is an interesting and inevitable topic for the transfer of high quality graphene onto other substrate materials. Our work clearly shows a strong improvement in the quality of graphene and therefore emphasizes the promising future of graphene as a virtually massless and ultrathin electrode material for high-frequency devices.

Acknowledgments: The authors would like to thank Michaela Fritz, Benedikt Tritschler and Andres Rösch for the technical and exploratory assistance and to acknowledge the funding from the European Union within the framework of Graphene Flagship.

Author Contributions: Marius Knapp, René Hoffmann and Oliver Ambacher conceived and designed the experiments; Marius Knapp performed the experiments and analyzed the data; René Hoffmann, Volker Cimalla and Oliver Ambacher contributed reagents/materials/analysis tools; Marius Knapp wrote the paper.

Conflicts of Interest: The authors declare no conflicts of interest. 


$\begin{array}{ll}\text { Abbreviations } & \\ \text { In this manuscript the following abbreviations are used: } \\ \text { RF } & \text { Radio frequency } \\ \text { MEMS } & \text { Microelectromechanical system } \\ \text { CVD } & \text { Chemical vapor deposition } \\ \text { AlN } & \text { Aluminum nitride } \\ \text { PMMA } & \text { Poly (methyl methacrylate) } \\ \text { BAW } & \text { Bulk acoustic wave } \\ \text { SMR } & \text { Solidly mounted resonator } \\ \text { AFM } & \text { Atomic force microscopy } \\ \text { CA } & \text { Contact angle }\end{array}$

\section{References}

1. Geim, A.K.; Novoselov, K.S. The rise of graphene. Nat. Mater. 2007, 6, 183-191. [CrossRef] [PubMed]

2. Lakin, K.M. A review of thin-film resonator technology. IEEE Microw. Mag. 2003, 4, 61-67. [CrossRef]

3. Bi, F.Z.; Barber, B.P. Bulk acoustic wave RF technology. IEEE Microw. Mag. 2008, 9, 65-80. [CrossRef]

4. Qian, Z.; Hui, Y.; Liu, F.; Kang, S.; Kar, S.; Rinaldi, M. Graphene-Aluminum nitride NEMS resonant infrared detector. Microsyst. Nanoeng. 2016, 2, 16026. [CrossRef]

5. Lee, S.; Adiga, V.P.; Barton, R.A.; Van Der Zande, A.M.; Lee, G.H.; Ilic, B.R.; Gondarenko, A.; Parpia, J.M.; Craighead, H.G.; Hone, J. Graphene metallization of high-stress silicon nitride resonators for electrical integration. Nano Lett. 2013, 13, 4275-4279. [CrossRef] [PubMed]

6. Jin, H.; Dong, S.R.; Luo, J.K.; Milne, W.I. Generalised Butterworth-Van Dyke equivalent circuit for thin-film bulk acoustic resonator. Electron. Lett. 2011, 47, 424. [CrossRef]

7. Thalhammer, R.; Fattinger, G.; Handtmann, M.; Marksteiner, S. Ohmic effects in BAW-resonators. In Proceedings of the 2006 IEEE MTT-S International Microwave Symposium Digest, San Francisco, CA, USA, 11-16 June 2006; pp. 7-10.

8. Miao, C.; Zheng, C.; Liang, O.; Xie, Y. Chemical Vapor Deposition of Graphene. In Physics and Applications of Graphene-Experiments; InTech: Rijeka, Croatia, 2011; p. 540. ISBN 978-953-307-217-3.

9. Suk, J.W.; Kitt, A.; Magnuson, C.W.; Hao, Y.; Ahmed, S.; An, J.; Swan, A.K.; Goldberg, B.B.; Ruoff, R.S. Transfer of CVD-grown monolayer graphene onto arbitrary substrates. ACS Nano 2011, 5, 6916-6924. [CrossRef] [PubMed]

10. Li, X.; Zhu, Y.; Cai, W.; Borysiak, M.; Han, B.; Chen, D.; Piner, R.D.; Colomba, L.; Ruoff, R.S. Transfer of large-area graphene films for high-performance transparent conductive electrodes. Nano Lett. 2009, 9 , 4359-4363. [CrossRef] [PubMed]

11. Meyer, J.; Kidambi, P.R.; Bayer, B.C.; Weijtens, C.; Kuhn, A.; Centeno, A.; Pesquera, A.; Zurutuza, A.; Robertson, J.; Hofmann, S. Metal Oxide Induced Charge Transfer Doping and Band Alignment of Graphene Electrodes for Efficient Organic Light Emitting Diodes. Sci. Rep. 2015, 4, 5380. [CrossRef] [PubMed]

12. Graphenea Inc. Logo. Available online: https://www.graphenea.com/collections/buy-graphene-films (accessed on 20 July 2017).

13. Qian, Z.; Liu, F.; Hui, Y.; Kar, S.; Rinaldi, M. Graphene as a Massless Electrode for Ultrahigh-Frequency Piezoelectric Nanoelectromechanical Systems. Nano Lett. 2015, 15, 4599-4604. [CrossRef] [PubMed]

14. Gun Oh, J.; Ki Hong, S.; Kim, C.K.; Hoon Bong, J.; Shin, J.; Choi, S.Y.; Jin Cho, B. High performance graphene field effect transistors on an aluminum nitride substrate with high surface phonon energy. Appl. Phys. Lett. 2014, 104, 193112. [CrossRef]

15. Chen, Y.; Gong, X.L.; Gai, J.G. Progress and Challenges in Transfer of Large-Area Graphene Films. Adv. Sci. 2016, 3, 1-15. [CrossRef] [PubMed]

16. Qian, Z.; Hui, Y.; Liu, F.; Kai, S.; Rinaldi, M. 1.27 GHz Graphene-Aluminum Nitride nano plate resonant infrared detector. In Proceedings of the 2015 Transducers-2015 18th International Conference on Solid-State Sensors, Actuators and Microsystems (TRANSDUCERS), Anchorage, AK, USA, 21-25 June 2015; pp. 1429-1432. [CrossRef] 
17. Gao, W.; Huang, R. Effect of surface roughness on adhesion of graphene membranes. J. Phys. D Appl. Phys. 2011, 44, 452001. [CrossRef]

18. Sönmez, T.; Fazeli Jadidi, M.; Kazmanli, K.; Birer, Ö.; Ürgen, M. Role of different plasma gases on the surface chemistry and wettability of RF plasma treated stainless steel. Vacuum 2016, 129, 63-73. [CrossRef]

19. Williams, D.L.; Kuhn, A.T.; Amann, M.A.; Hausinger, M.B.; Konarik, M.M.; Nesselrode, E.I. Computerised measurement of contact angles. Galvanotechnik 2010, 101, 2502-2512.

20. Quéré, D. Wetting and Roughness. Annu. Rev. Mater. Res. 2008, 38, 71-99. [CrossRef]

21. Seo, K.; Kim, M.; Kim, D.H. Re-derivation of Young's Equation, Wenzel Equation, and Cassie-Baxter Equation Based on Energy Minimization. Surf. Energy 2015. [CrossRef]

22. Prysiazhnyi, V. Atmospheric Pressure Plasma Treatment and Following Aging Effect of Chromium Surfaces. J. Surf. Eng. Mater. Adv. Technol. 2013, 3, 138-145. [CrossRef]

23. Lee, J.E.; Ahn, G.; Shim, J.; Lee, Y.S.; Ryu, S. Optical separation of mechanical strain from charge doping in graphene. Nat. Commun. 2012, 3, 1024-1028. [CrossRef] [PubMed]

24. Das, A.; Chakraborty, B.; Piscanec, S.; Pisana, S.; Sood, A.K.; Ferrari, A.C. Phonon renormalization in doped bilayer graphene. Phys. Rev. B Condens. Matter Mater. Phys. 2009, 79, 155417. [CrossRef]

25. Das, A.; Pisana, S.; Piscanec, S.; Chakraborty, B.; Saha, S.K.; Waghmare, U.V.; Yiang, R.; Krishnamurhthy, H.R.; Geim, A.K.; Ferrari, A.C.; et al. Electrochemically Top Gated Graphene: Monitoring Dopants by Raman Scattering. Nat. Nanotechnol. 2007, 3, 210-215. [CrossRef] [PubMed]

26. Yoon, D.; Son, Y.W.; Cheong, H. Strain-dependent splitting of the double-resonance raman scattering band in graphene. Phys. Rev. Lett. 2011, 106, 1-4. [CrossRef] [PubMed]

27. Ni, Z.; Wang, Y.; Yu, T.; Shen, Z. Raman spectroscopy and imaging of graphene. Nano Res. 2010, 1, $273-291$. [CrossRef]

28. Wang, Q.J.; Chung, Y.-W. Encyclopedia of Tribology, 1st ed.; Springer International Publishing: Basel, Switzerland, 2013; ISBN 9780387928982.

29. Li, X.; Cai, W.; An, J.; Kim, S.; Nah, J.; Yang, D.; Piner, R.; Velamakanni, A.; Jung, I.; Tutuc, E.; et al. Large-area synthesis of high-quality and uniform graphene films on copper foils. Science 2009, 324, 1312-1314. [CrossRef] [PubMed]

30. Ferrari, A.C.; Meyer, J.C.; Scardaci, V.; Casiraghi, C.; Lazzeri, M.; Mauri, F.; Piscanec, S.; Jiang, D.; Novoselov, K.S.; Roth, S.; et al. Raman Spectrum of Graphene and Graphene Layers. Phys. Rev. Lett. 2006, 97, 187401. [CrossRef] [PubMed]

31. Malard, L.M.; Pimenta, M.A.; Dresselhaus, G.; Dresselhaus, M.S. Raman spectroscopy in graphene. Phys. Rep. 2009, 473, 51-87. [CrossRef]

32. Wu, Z.T.; Zhao, W.W.; Chen, W.Y.; Jiang, J.; Nan, H.Y.; Guo, X.T.; Liang, Z.; Chen, Y.M.; Chen, Y.F.; Ni, Z.H. The influence of chemical solvents on the properties of CVD graphene. J. Raman Spectrosc. 2015, 46, 21-24. [CrossRef]

33. Shang, J.; Flury, M.; Harsh, J.B.; Zollars, R.L. Comparison of different methods to measure contact angles of soil colloids. J. Colloid Interface Sci. 2008, 328, 299-307. [CrossRef] [PubMed]

(C) 2017 by the authors. Licensee MDPI, Basel, Switzerland. This article is an open access article distributed under the terms and conditions of the Creative Commons Attribution (CC BY) license (http://creativecommons.org/licenses/by/4.0/). 